\title{
Bufalin induces G2/M phase arrest and triggers autophagy via the TNF, JNK, BECN-1 and ATG8 pathway in human hepatoma cells
}

\author{
CHIN-MU HSU ${ }^{1-3}$, YUHSIN TSAI $^{1,2}$ LEI WAN $^{1-3^{*}}$ and FUU-JEN TSAI ${ }^{2,4,5^{*}}$ \\ ${ }^{1}$ Graduate Institute of Chinese Medicine, ${ }^{2}$ School of Chinese Medicine, China Medical University; \\ Departments of ${ }^{3}$ Medical Research and ${ }^{4}$ Medical Genetics, Pediatrics and Medical Research, \\ China Medical University Hospital, 40402 Taichung; ${ }^{5}$ Department of Biotechnology, \\ Asia University, 41354 Taichung, Taiwan, R.O.C.
}

Received January 16, 2013; Accepted March 18, 2013

DOI: $10.3892 /$ ijo.2013.1942

\begin{abstract}
Liver cancer is the fifth most common cause of cancer death worldwide. The study of more effective anti-hepatoma drugs is urgently required. Bufalin has been isolated from a traditional Chinese medicine and possesses less toxicity to normal cells. However, it has been found to inhibit growth of cancer cells. In this study, we aimed to investigate the efficacy and mechanism of bufalin in Huh7, Hep3B and HA22T human hepatoma cells. The three cell lines were treated with bufalin, the proliferation was detected by WST-1 assay and cell cycle was detected by flow cytometry analysis. The results showed that bufalin inhibited the proliferation of hepatoma cells and regulated the hepatoma cell death program in a dose- and time-dependent manner without typical features of apoptosis. RT-PCR arrays were used to investigate the autophagy transcriptional response triggered by bufalin and 13 genes were altered and further confirmed by real-time PCR. The translation levels of selected genes were examined by western blot analysis to reveal the bufalin-induced autophagy cascade. Bufalin synergized with the JNK pathway to induce autophagy of hepatoma cells and is closely associated with the upregulation of TNF, BECN-1, MAPK and ATG8, together with the downregulation of Bcl-2 and Bid. Our study provided a multi-angle evaluation system
\end{abstract}

Correspondence to: Professor Fuu-Jen Tsai, Department of Medical Genetics, Pediatrics and Medical Research, China Medical University Hospital, 2 Yuh-Der Road, 40402 Taichung, Taiwan, R.O.C.

E-mail: d0704@mail.cmuh.org.tw

Professor Lei Wan, Graduate Institute of Chinese Medicine, China Medical University, 91 Hsueh-Shih Road, 40402 Taichung, Taiwan, R.O.C.

E-mail: leiwan@mail.cmu.edu.tw

*Contributed equally

Abbreviations: Bid, BH3 interacting-domain death agonist; Bax, Bcl-2-associated X protein; Bcl-2, B-cell lymphoma 2

Key words: bufalin, autophagy, hepatoma cell, G2/M phase arrest, PCR array for anti-hepatoma pharmacology for pre-clinical drug investigation. In this case, bufalin was capable of inducing hepatoma cell autophagy, suggesting a potential regimen for single or combined chemotherapy to overcome hepatoma in clinical practice.

\section{Introduction}

Liver cancer shows high incident and mortality all over the world. It ranks the fifth and seventh most diagnosed cancer worldwide for male and female, respectively (1), and is the second and sixth leading cause of cancer death for man and women, respectively (1). In 2008, almost 750,000 new cases were recorded and 700,000 cancer deaths occurred worldwide (1). Geographically, the regions of highest liver cancer rates in the world are East and South-East Asia, Middle and Western Africa $(1,2)$. In America and Europe, over the past 10 years, incidence rates of liver cancer among men and women increased and cancer mortality trends (death rates) increased for liver cancer in all age groups and among white, black and Hispanic men and white women (3). The differential trend is found in Japan, where the incidence and mortality of liver cancer is declining, indicated that there may be a difference in Eastern and Western countries (4). This may be due to variations in genetic background, environmental exposure, diet habits and hepatitis B virus (HBV, for the Eastern) or hepatitis $\mathrm{C}$ virus (HCV, for USA and Europe) infection (2).

The therapeutic strategies of liver cancer include surgical treatments and chemotherapy. Radiation is not considered since the liver is the largest organ with the endocrinic and detoxicificative functions. Liver surgical treatments are resection, liver transplantation and chemoembolization. In chemotherapy for liver cancer, the commonly used drugs include cisplatin, epirubicin, etoposide and 5-fluorouracil, all have an unsatisfactory therapeutic efficacy in single drug treatment (5). The most promising agent for treatment of liver cancer is the oral multikinase inhibitor, sorafenib. However, it is reported that its efficacy in cancer cell killing is not satisfactory enough without the combination of other drugs, such as fluvastatin (6). Therefore, a more effective anticancer drug is urgently needed for its development and revealing its efficacy mechanism. 
Bufalin, a bufadienolide derivative, is the active compound of cinobufacini (Fig. 1). In literature, cinobufacini is water soluble and extracted from the dried toad skin of traditional Chinese medicine with a variety of biological activities $(7,8)$. Bufalin is effective in cardiotonic and anaesthetic treatment, blood pressure controlling and promoting antineoplastic activities (9). In addition, bufalin was reported to exhibit significant antitumor activity in hepatocellular carcinoma, non-small cell lung cancer, pancreatic cancer and gallbladder carcinoma, with low toxicity and few side effects $(8,10,11)$. Moreover, bufalin can inhibit cell growth and proliferation in various human cells, including colon cancer, hepatoma, leukemia, endometrial cancer and ovarian cancer (12-15). However, the detail mechanism is not well understood.

Autophagy is a mechanism of cell suicide which eliminates malignant cells, supports the surviving cells promoting the viability of the whole population. In programmed cell death, the process of autophagy is regulated by multiple autophagyrelated (Atg) genes and correlated with cell cycle arrest (16). Mounting evidence is revealing the pathways responsible for autophagy in different cells under various environmental stresses (17). Only one report exists on investigation of the mechanism of bufalin-induced autophagy, which reported that reactive oxygen species (ROS) and c-Jun $\mathrm{NH}_{2}$-terminal kinase (JNK) pathways were involved (12). In addition to apoptosis, autophagy is a novel cell death type in cancer cell killing for potential anticancer drug candidates $(18,19)$. It is more beneficial for the anticancer drugs to induce autophagy rather than apoptosis in the cancer patients and the prognosis may be better in the patients treated by drugs with the former efficacy.

In this study, we examined the chemotherapeutic efficacy of bufalin in inhibition of the proliferation in human hepatoma cell lines, Huh7, Hep3B and HA22T. Our results unveiled that bufalin could arrest cell cycle at G2/M phase and induce cell death by autophagy instead of apoptosis in hepatoma cells.

\section{Materials and methods}

Cell culture. Three hepatoma cancer cell lines, Huh7, Hep3B and HA22T, were obtained from the Bioresource Collection and Research Center (BCRC, Taiwan) and cultured in DMEM with $10 \%$ fetal bovine serum (FBS) and $1 \%$ antibiotics (penicillin/streptomycin; PS; Gibco). Culture medium was replenished every 3-4 days and grown at $37^{\circ} \mathrm{C}$ in a humidified atmosphere containing $5 \% \mathrm{CO}_{2}$. Bufalin was purchased from Phytomarker Ltd. (Tianjin, China) and was dissolved in DMSO and maintained at $-20^{\circ} \mathrm{C}$ as a $20 \mathrm{mM}$ stock.

Cell viability and cell death assay. For cell viability assay, the cells were seeded in 96-well plates at a density of 3,000 cells/well overnight, treated with the respective agents (bufalin) for $72 \mathrm{~h}$ and then exposed to WST-1 reagent (Roche, Mannheim, Germany) for $4 \mathrm{~h}$ at $37^{\circ} \mathrm{C}$ according to the manufacturer's instructions. Absorbance was measured at $450 \mathrm{~nm}$ on a microplate Reader (iMark ${ }^{\mathrm{TM}}$ Microplate Absorbance Reader, Bio-Rad Laboratories, Hercules, CA, USA). Cell viability was also evaluated by counting cells that excluded trypan blue. All experiments were done at least three times.

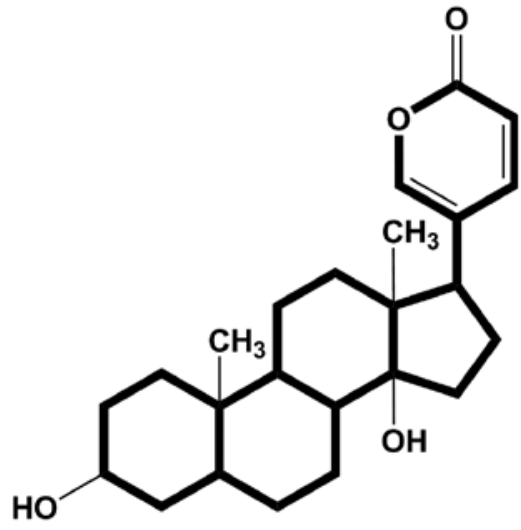

Figure 1. Molecular structure of bufalin.

Analysis of cell cycle by flow cytometry. The cell cycle analysis by flow cytometry as previous descripted after $24 \mathrm{~h}$ of culture either with or without bufalin (20). Briefly, Huh7, Hep3B and $\mathrm{HA} 22 \mathrm{~T}$ cells were cultured at $1 \mathrm{x} 0^{6}$ cells for different time courses with or without the presence of bufalin $(0.04 \mu \mathrm{M})$. Then the cells were trypsinized, washed in PBS, fixed in $70 \%$ methanol and incubated for $30 \mathrm{~min}$ at $4^{\circ} \mathrm{C}$ in the dark with a PBS solution of $5 \mu \mathrm{g} / \mathrm{ml}$ propidium iodide (Sigma), $1 \mathrm{mg} / \mathrm{ml}$ RNase (Sigma) and 0.1\% Nonidet P-40 (Sigma). Stained cells were immediately analyzed using a FACSCanto flow cytometry system (BD Biosciences, San Jose, CA). Flow cytometry analysis of the cell cycle was performed immediately using the ModFit LT 3.0 program (Verity Software House, Inc., ME).

Gene expression profiling by PCR array. To examine the effects of bufalin treatment on gene expression in hepatoma cells, Huh7 cells were treated with bufalin $(0.04 \mu \mathrm{M})$ for $12 \mathrm{~h}$. At the termination of an experiment, total RNA was extracted by using an RNeasy Mini kit (Qiagen, Valencia, CA) and processed for PCR array analysis. Quantification and quality control of total RNAs have been performed by the measurement of optical densities at 260 and $280 \mathrm{~nm}$. Reverse transcriptions have been performed on $1 \mu \mathrm{g}$ of total RNA in a 20- $\mu$ l final volume using the High-Capacity cDNA Reverse Transcription kit (Applied Biosystems, CA, USA). Expression of genes involved in the bufalin treatment was studied by using 96-well RT ${ }^{2}$ Profiler PCR Arrays-Human Autophagy (Qiagen, Frederick, MD, USA) in a LightCycler 480 PCR system (Roche, Germany).

Quantitative real-time PCR. Relative real-time PCR was performed on a LightCycler 480 PCR system (Roche, Germany) with gene-specific primers and TaqMan probes protocol to confirm the gene expression changes observed by using PCR array. Total RNA ( $2 \mu \mathrm{g})$ from each pool was reverse transcribed to cDNA in the presence of random primer sequences in total volume of $20 \mu \mathrm{l}$. After dilution of the cDNA with $80 \mu \mathrm{l}$ of water, $2 \mu \mathrm{l}$ of this cDNA was used as template in the real-time PCR. Relative expression ratios were normalized to glyceraldehydes 3-phosphate dehydrogenase (GAPDH). The PCR primers used in this study are available upon request and in Table III. All PCRs were performed in triplicates. 


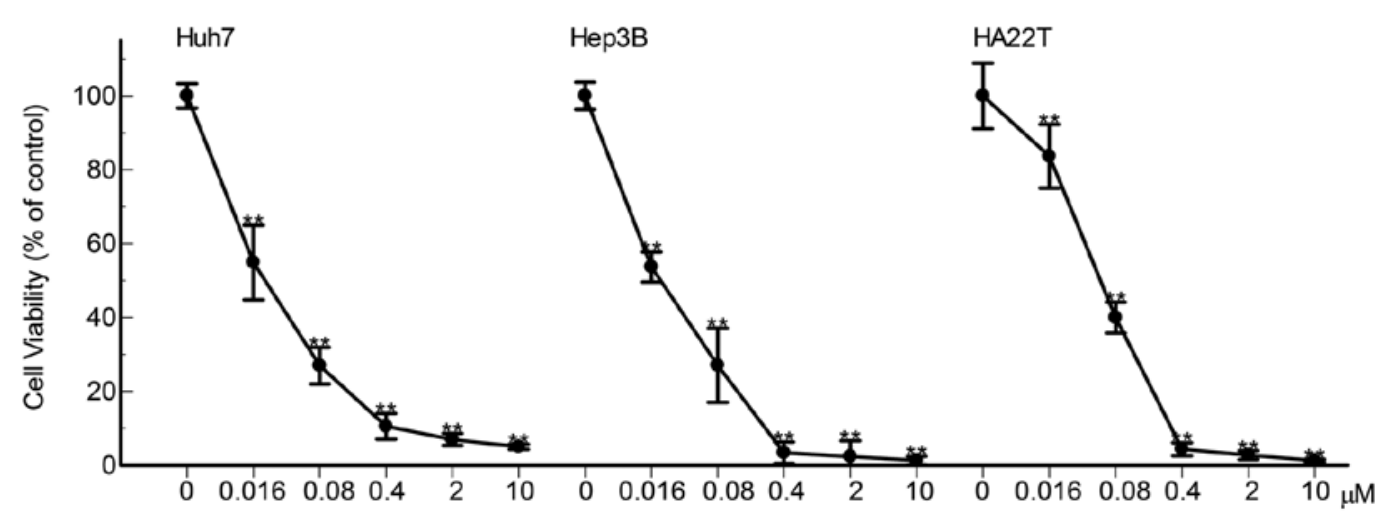

Figure 2. Bufalin-induced cytotoxicity in hepatoma cells. Cells were seeded in 96-well plates $24 \mathrm{~h}$ before treatment and Huh7, Hep3B and HA22T cells were treated with bufalin at indicated doses. Cells were harvested after 72-h treatment and WST-1 assays were performed immediately. The percentage of cell viability is shown for each treatment compared to the sham-treated cells, which were considered as $100 \%$ control. Experiments were conducted in triplicate.

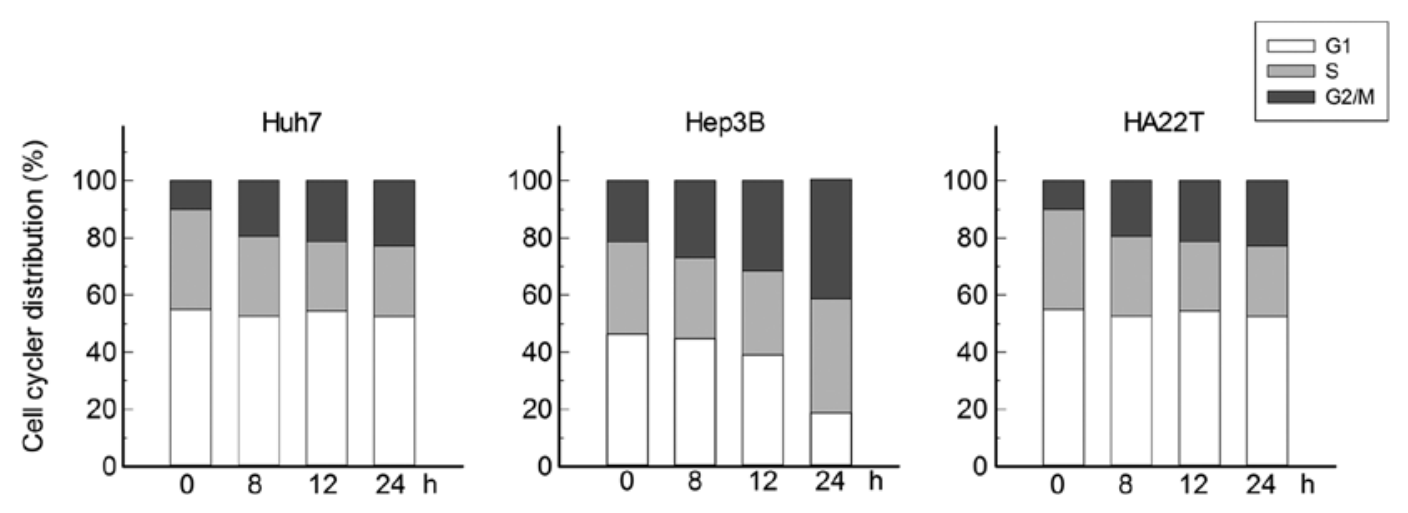

Figure 3. The G2/M phase cell cycle arrest induced by bufalin in hepatoma cells at 0, 8, 12 and 24 h post-treatment. Huh7, Hep3B and HA22T cells were collected at $0,8,12$ and $24 \mathrm{~h}$ after $0.04 \mu \mathrm{M}$ bufalin treatment and subjected to flow cytometry. Percentages of cells distributed in the G1, S and G2/M phases of the cell cycle were determined by Mitfit LC 3.0 .

Western blot analysis. Cells were treated with bufalin $(0.04 \mu \mathrm{M})$ for $0,4,8$ and $12 \mathrm{~h}$. After treatment, total cell lysates were prepared and $30 \mu \mathrm{g}$ protein was subjected to sodium dodecyl sulfate polyacrylamide gel electrophoresis (SDS-PAGE), followed by immunoblot analysis. Primary antibodies used included anti-PARP, cytochrome C, cdc25c, cyclin B and cdc2/cdk1 (Cell Signaling, Beverly, MA); Bid (BD Biosciences, San Diego, CA) and GABARAPL1 (GeneTex, San Antonio, TX). Anti-rabbit or anti-mouse secondary antibody conjugated with horseradish peroxidase was also used (GE Healthcare, Piscataway, NJ). Immunoreactive bands were detected by enhanced chemiluminescence kit (ECL, Pierce, Thermo Fisher Scientific, Pittsburgh, PA, USA) for western blotting detection by using a ChemiGenius bioimaging system (Syngene, USA). Equal loading was confirmed via probing the blots with $\beta$-actin antibody (Abcam, Cambridge, MA, USA).

Statistical analysis. Statistical analysis was performed using Student's t-test for comparison of two groups or one-way analysis of variance for comparison of more than two groups followed by Tukey's multiple comparison test. Statistical calculations were performed using the software from SPSS (SPSS Institute, Chicago, IL, USA). Data were expressed as means \pm SEM of at least three independent experiments. A $\mathrm{p}<0.05$ was considered statistically significant.

\section{Results}

Effects of bufalin on the proliferation and viability of human hepatic cell lines in vitro. To investigate the anticancer efficacy of bufalin on three hepatoma cell lines, Huh7, Hep3B and HA22T cells were treated with physiological achievable concentrations of bufalin for $72 \mathrm{~h}$. As shown in Fig. 2, the overall cytotoxicity of bufalin in Huh7, Hep3B and HA22T cells are presented and the growth of Huh7, Hep3B and HA22T were inhibited by bufalin dose-dependently. The absent of floating cells may indicate that there were no dying cells at these concentrations. Approximately $95 \%$ of cell proliferation of the three cell lines was inhibited by bufalin at 10,2 and as low as $0.4 \mu \mathrm{M}$ after 72 -h bufalin treatment. The $\mathrm{IC}_{50}$ values were 0.034-0.04 $\mu \mathrm{M}$ for each cell line (Fig. 2). The results suggested that the micromolar level of bufalin can inhibit cell proliferation of hepatoma cells.

Bufalin arrested the cell cycle of hepatoma cells at G2/M phase. To further investigate the effect of bufalin on the cell cycle regulation of hepatoma cells, Huh7, Hep3B and HA22T cells was treated with $0.04 \mu \mathrm{M}$ of bufalin for $0,8,12$ and $24 \mathrm{~h}$. Then the cells were collected and fixed with $70 \%$ methanol overnight and then stained with PI to detect the distribution of cell cycle by flow cytometry. As shown in Fig. 3, hepatoma 


\begin{tabular}{llllll}
\hline & 4 & 4 & 8 & 12 & $h$
\end{tabular}
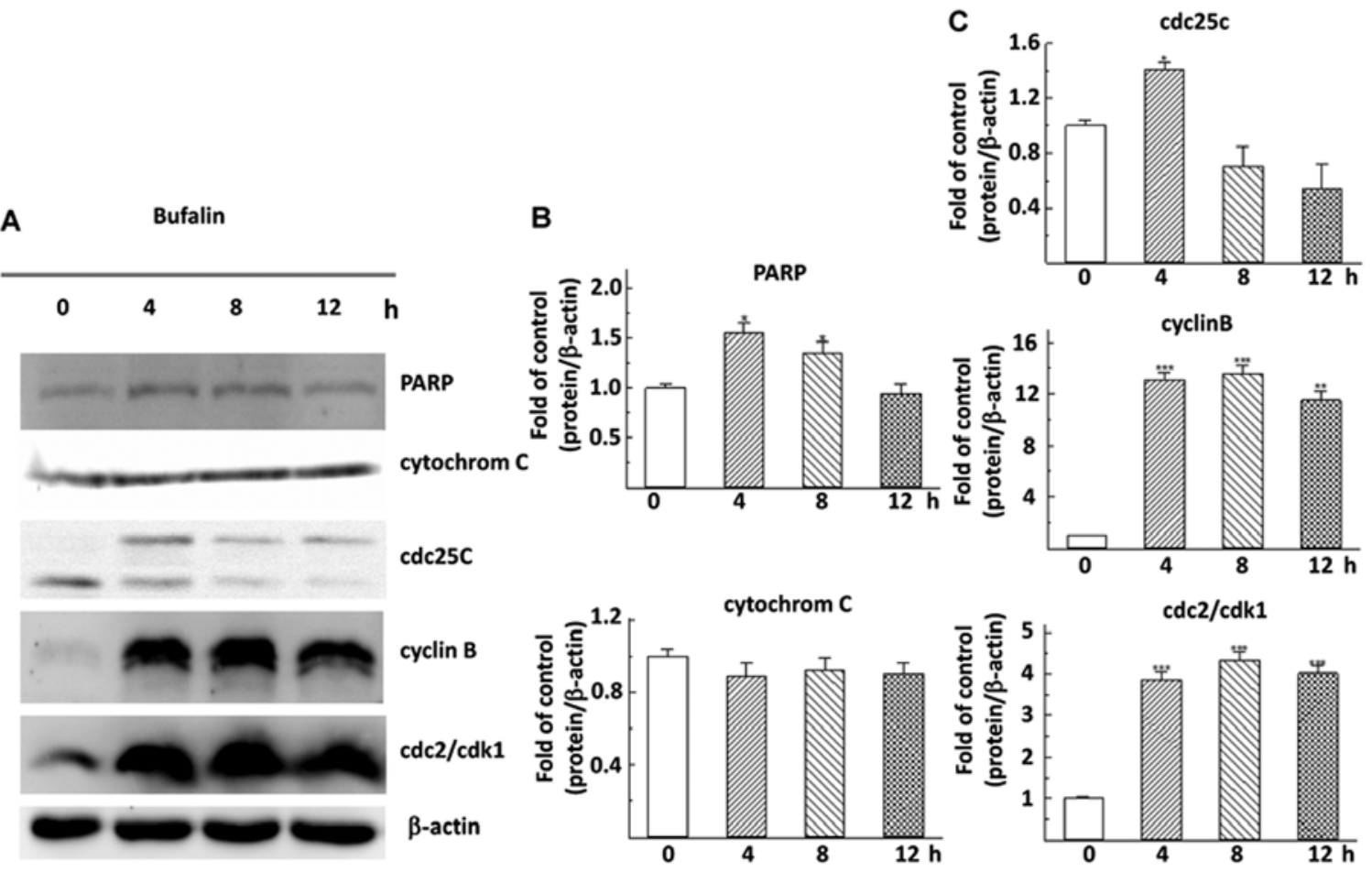

Figure 4. Bufalin affected the expression levels of G2/M phase cell cycle-associated proteins but not apoptosis-associated proteins in Huh7 cells. (A) Cells were exposed to $0.04 \mu \mathrm{M}$ bufalin for $0,4,8$ and $12 \mathrm{~h}$ and then harvested and lysed for the detection of protein levels of PARP, cytochrome C, cdc25c, cyclin B and cdc2 by western blot analysis. $\beta$-actin was used as an internal control. (B) Quantitative analysis of expression of PARP and cytochrome C ( $\mathrm{n}=3$ in each group). (C) Quantitative analysis of expression of cdc25c, cyclin $\mathrm{B}$ and cdc2 ( $\mathrm{n}=3$ in each group, ${ }^{*} \mathrm{P}<0.05 ;{ }^{* *} \mathrm{P}<0.01 ;{ }^{* * * *} \mathrm{P}<0.001$ compared with internal group).

cells were increased at the $\mathrm{G} 2 / \mathrm{M}$ phase by $0.04 \mu \mathrm{M}$ of bufalin time-dependently, with a concomitant decrease in the proportion of those in the S and G1 phase (Fig. 3).

Effects of bufalin on the expression level of G2/M phaserelated and apoptosis-related proteins in hepatoma cells. To investigate if the arrest in G2/M phase of cell cycle and the relationship between cell cycle, apoptosis and autophagy, we evaluated the expression of G2/M phase of cell cycle-related and apoptosis-related proteins in hepatoma cells. In Fig. 4 (A and C), bufalin time-dependently upregulated the expression of the cyclin B, cdc2 and cdc $25 \mathrm{c}$ protein, the check-point of G2/M phase of cell cycle, in hepatoma cells (Fig. 4A and 4C). At the same time, the levels of cytochrome C and PARP proteins, which closely associated with apoptosis in hepatoma cells, were unchanged after bufalin treatment (Fig. 4B).

Effects of bufalin on autophagy-associated proteins at transcriptional level by PCR array analysis. To assess the overall gene expression alteration pattern by bufalin in the hepatoma cells, differential patterns of sham-treated and $0.04 \mu \mathrm{M}$ bufalin-treated Huh7 cells were compared by a cDNA PCR array. Samples were processed and up to 84 of annotated human cDNAs were analyzed and compared in the array system. Table I shows that 14 of the 84 detected genes were significantly altered by 2 -fold (either up- or downregulated) after $0.04 \mu \mathrm{M}$ bufalin treatment for $12 \mathrm{~h}$ in the Huh7 cells. The genes encoding co-regulators of autophagy and apoptosis (CXCR4, TNF, IFNG, IFNA2,
PIK3CG), autophagy induction by intracellular pathogens (IFNA2) and co-regulators of autophagy and the cell cycle (IFNG) were upregulated. The expression level of GABARAPL1 and RPS6KB1, which encoded an autophagic vacuole formation and autophagy in response to other intracellular signals, respectively, were also increased. On the contrary, the translational level of the other 7 genes was significantly downregulated. Four main subgroups could be identified: genes encoding co-regulators of autophagy and apoptosis (IGF1, BID, TNFSF10), chaperone-mediated autophagy (HSP90AA1, HSPA8), autophagic vacuole formation (RGS19) and autophagy in response to other intracellular signals (ULK2).

Confirmation of the PCR array data with real-time PCR of the transcriptional changes for a selected subset of 14 genes in Huh7 cells. A subset of Huh7 genes potentially involved in autophagy was selected for further analysis by real-time PCR to confirm and to more precisely quantify the changes at the mRNA expression levels. As shown in Table II, these data synchronized with the results of the real-time PCR array analysis; the upregulated genes of CXCR4, 13.61x; TNF, 17.43x; IFNG, 6.96x; IFNA2, 3.83x; GABARAPL1, 3.43x and RPS6KB1, 1.35x; and repressed genes of HSPA8, HSP90AA1, BID, RGS19, ULK2 and TNFSF10 (HSPA8, 16.03x; HSP90AA1, 1.61x; BID, 1.3x; RGS19, 2.41x; ULK2, 4.18x and TNFSF10, 8.55x repression) were confirmed. IGF1 mRNA level was not altered and the transcriptional level of PIK3CG was undetectable. 
Table I. Gene expression pattern modulated by bufalin in Huh7 cells.

\begin{tabular}{|c|c|c|}
\hline Gene name/symbol & Fold alternation & Description \\
\hline \multirow{2}{*}{\multicolumn{3}{|c|}{$\begin{array}{l}\text { Co-regulators of autophagy } \\
\text { and apoptosis }\end{array}$}} \\
\hline & & \\
\hline CXCR4 & 11.63 & Chemokine (C-X-C motif) receptor 4 \\
\hline TNF & 7.67 & Tumor necrosis factor \\
\hline IFNG & 4.72 & Interferon $\gamma$ \\
\hline IFNA2 & 3.51 & Interferon $\alpha 2$ \\
\hline PIK3CG & 2.23 & Phosphoinositide-3-kinase, catalytic, $\gamma$ polypeptide \\
\hline IGF1 & -2.00 & Insulin-like growth factor 1 (somatomedin C) \\
\hline BID & -2.03 & $\mathrm{BH} 3$ interacting domain death agonist \\
\hline TNFSF10 & -4.69 & Tumor necrosis factor (ligand) superfamily, member 10 \\
\hline \multicolumn{3}{|c|}{ Genes involved in autophagic } \\
\hline \multicolumn{3}{|c|}{ vacuole formation } \\
\hline GABARAPL1 & 2.64 & GABA(A) receptor-associated protein like 1 \\
\hline RGS19 & -2.07 & Regulator of G-protein signaling 19 \\
\hline \multicolumn{3}{|c|}{ Chaperone-mediated autophagy } \\
\hline HSP90AA1 & -2.75 & Heat shock protein $90-\mathrm{kDa} \alpha$ (cytosolic), class A member 1 \\
\hline HSPA8 & -10.70 & Heat shock $70-\mathrm{kDa}$ protein 8 \\
\hline \multicolumn{3}{|c|}{$\begin{array}{l}\text { Autophagy induction by intracellular } \\
\text { pathogens }\end{array}$} \\
\hline IFNA2 & 3.51 & Interferon $\alpha 2$ \\
\hline \multicolumn{3}{|c|}{$\begin{array}{l}\text { Co-regulators of autophagy and the } \\
\text { cell cycle }\end{array}$} \\
\hline IFNG & 4.72 & Interferon $\gamma$ \\
\hline \multicolumn{3}{|c|}{ Autophagy in response to other } \\
\hline \multicolumn{3}{|l|}{ intracellular signals } \\
\hline ULK2 & -2.50 & Unc-51-like kinase 2 (C. elegans) \\
\hline \multicolumn{3}{|c|}{$\begin{array}{l}\text { Autophagy in response to other } \\
\text { intracellular signals }\end{array}$} \\
\hline RPS6KB1 & 2.08 & Ribosomal protein S6 kinase, 70-kDa, polypeptide 1 \\
\hline
\end{tabular}

Time course of bufalin-regulated gene expression in three hepatoma cells. A 2-fold alteration in expression was defined as the minimum cut-off for the significant alteration in PCR array analysis; we selected 13 genes (Table III) for further analysis to see the time-course differences of the transcription level in Huh7, Hep3B and HA22T cells after bufalin treated. We found 7 genes to be upregulated in their expression at 4, 8 and $12 \mathrm{~h}$ in Huh7 cells. Moreover, the transcriptional level of ATG8, which was reported to closely relate to autophagy, was activated in all the three cell lines time-dependently. In contrast, six genes were found to be decreased at their mRNA expression levels at 4,8 and $12 \mathrm{~h}$ in the three cell types. The results of quantitative real-time PCR analysis for all the 13 selected genes functionally related to hepatoma cell autophagy were in agreement with the PCR array findings for each gene detected (Fig. 5).

Bufalin inhibits BID and activates GABARAP (ATG8) family proteins in Huh7 cells. To examine whether BID and GABARAP family proteins were involved in bufalin-induced autophagy in hepatoma cells as reported in other cancer cells, the effects of bufalin on their expression levels were detected by western blot assay in Huh7 cells. Cells were harvested at different times after treatment with $0.04 \mu \mathrm{M}$ bufalin and then 
Table II. The synchronization of PCR array and real-time PCR in Huh7 cell after 12-h bufalin treatment.

\begin{tabular}{lccl}
\hline & \multicolumn{2}{c}{ Fold alternation } & \\
\cline { 2 - 3 } Gene name & PCR array & RT-PCR & Synchronize \\
\hline CXCR4 & 11.63 & $13.61 \pm 4.84$ & Both positive \\
TNF & 7.67 & $17.43 \pm 2.83$ & Both positive \\
IFNG & 4.72 & $6.96 \pm 3.63$ & Both positive \\
IFNA2 & 3.51 & $3.83 \pm 1.42$ & Both positive \\
GABARAPL1 & 2.64 & $3.43 \pm 1.05$ & Both positive \\
PIK3CG & 2.23 & No on & Only PCR \\
& & QRT-PCR & array \\
RPS6KB1 & 2.08 & $1.35 \pm 0.26$ & Both positive \\
IGF1 & -2 & $4.24 \pm 1.89$ & Not altered \\
BID & -2.03 & $-1.30 \pm 0.27$ & Both negative \\
RGS19 & -2.07 & $-2.41 \pm 0.19$ & Both negative \\
ULK2 & -2.5 & $-4.18 \pm 0.11$ & Both negative \\
HSP90AA1 & -2.75 & $-1.61 \pm 0.63$ & Both negative \\
TNFSF10 & -4.69 & $-8.55 \pm 0.01$ & Both negative \\
HSPA8 & -10.7 & $-16.08 \pm 0.01$ & Both negative \\
\hline
\end{tabular}

Real-time PCR analysis of the expression of the selected set of genes closely associated with autophagy. Real-time PCR analysis was used to confirm and further quantity the expression changes showed in the PCR array experiment (Table I). The abbreviated gene names are listed in the first column; second column displays the alternated folds after bufalin 12-h treatment compared with sham-treated control group based on the microarray experiment; third column shows the alternated folds based on real-time PCR and the last column presented the synchronized results of PCR array and real-time PCR.

determined the BID and GABARAP family-related protein levels by western blotting. The results shown in Fig. 6 revealed that bufalin decreased BID after 12-h treatment. The data also showed that bufalin increased the levels of GABARAP family proteins (GABARAP and GABARAPL1) from 4- to 8- and 12-h treatment in Huh7 cells (Fig. 6). Based on these findings, it was suggested that bufalin-induced autophagy in Huh7 cells was mainly mediated through the downregulation of BID and upregulation of GABARAP family proteins.

\section{Discussion}

Bufalin has been demonstrated to have therapeutic effect in cancer cells through apoptosis mechanisms, but the signaling pathways of autophagy underlying bufalin-induced cell death in hepatoma cells have not been elucidated (21). In this study, we examined the effects of bufalin on hepatoma cell lines and aimed at unveiling the inhibition of cell growth with bufalin treatment and the molecular mechanism of bufalin-induced cell death in hepatoma cells. At the dosage of $0.4 \mu \mathrm{M}$ or above, bufalin was effective in decreasing the percentage of cell viability for all the three hepatoma cell lines examined, Huh7, Hep3B and HA22T cells, to $<10 \%$. Also, the calculated $\mathrm{IC}_{50}$ was as low as $0.04 \mu \mathrm{M}$ in these examined cells, strength-

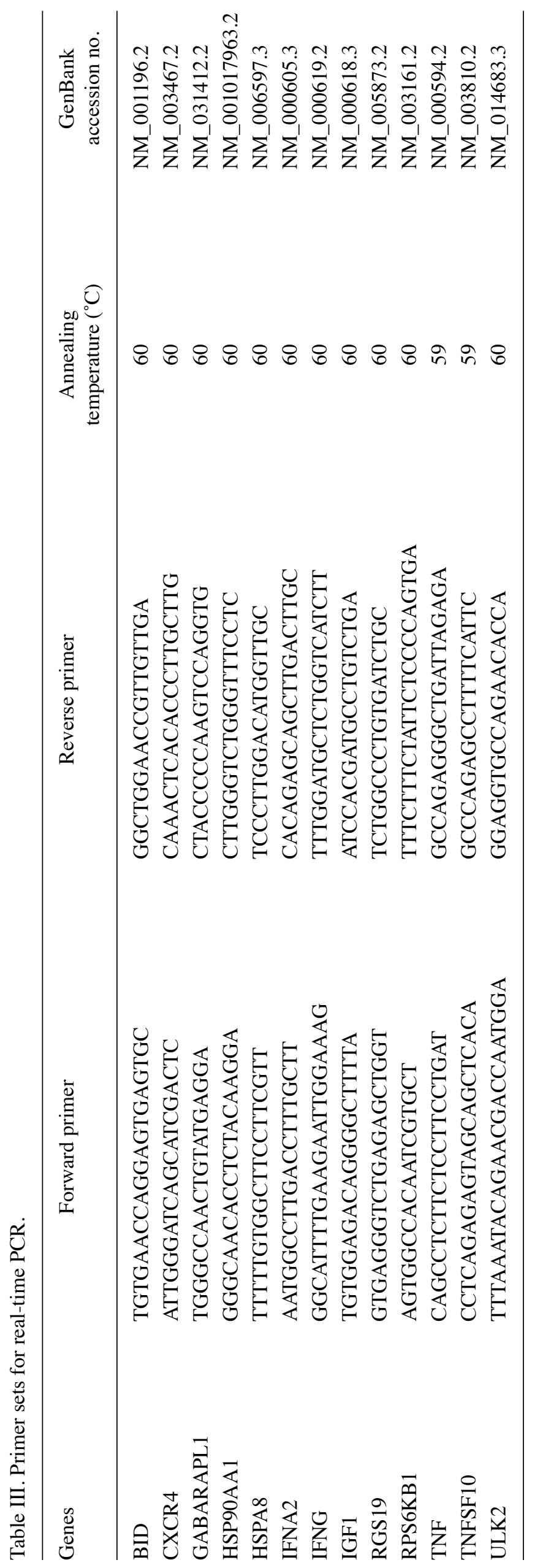



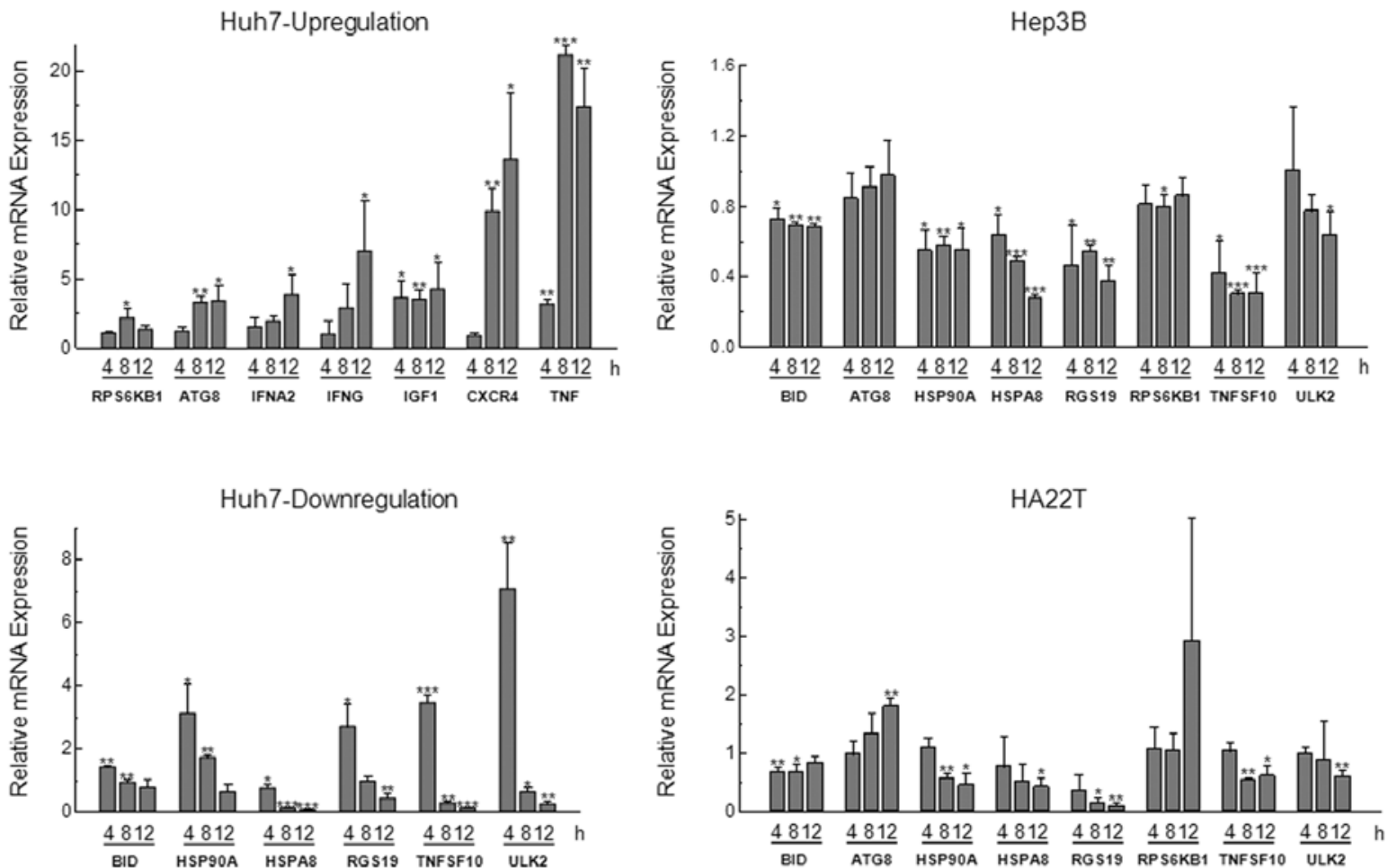

Figure 5. Confirmation of the PCR array data with real-time PCR of the transcriptional changes for a selected subset of 14 genes in Huh7 cells. Real-time PCR was performed on cDNA prepared from RNA isolated from Huh7, Hep3B and HA22T cells at 4, 8 and $12 \mathrm{~h}$ after bufalin treatment using the TaqMan probe. GAPDH was used as an internal control. Results are representative of three independent experiments.

A
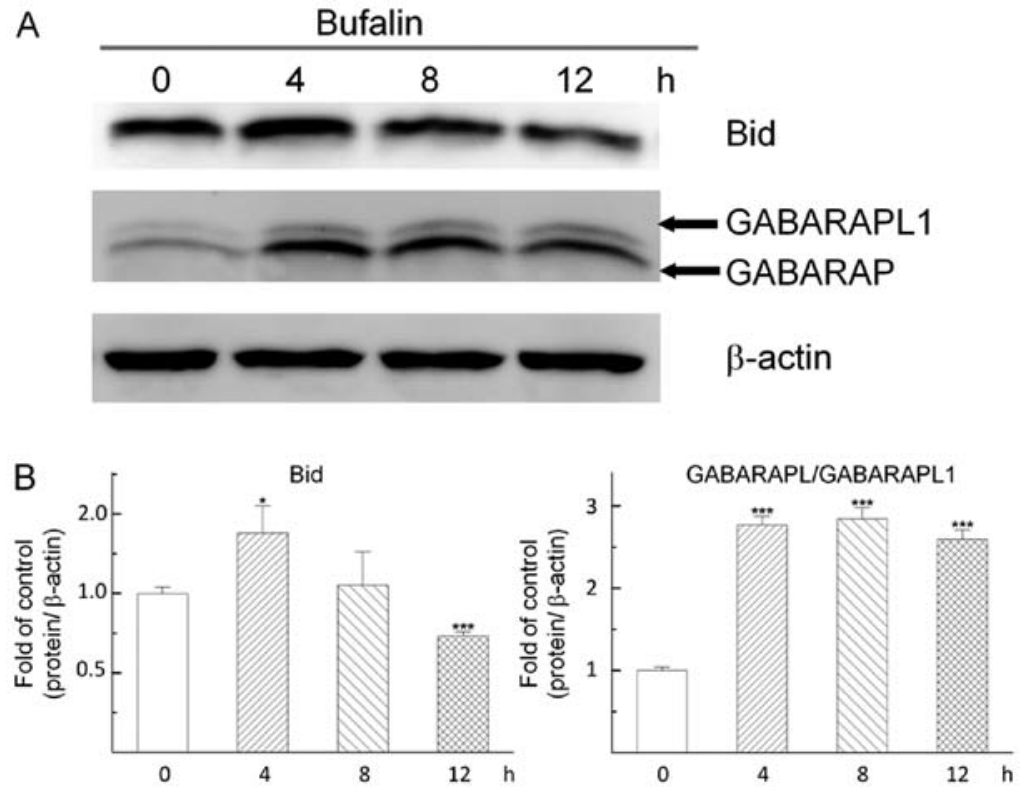

Figure 6. Bufalin induced autophagy related proteins in hepatoma cells. Western blot analysis of BID and GABARAPL1 expression in Huh7 cells treated with bufalin at $0.04 \mu \mathrm{M}$ for various time intervals. The amount of $\beta$-actin served as the loading control. Each image is representative of at least three independent experiments ( $\mathrm{n}=3$ in each group, ${ }^{*} \mathrm{P}<0.05 ;{ }^{* *} \mathrm{P}<0.01 ;{ }^{* * *} \mathrm{P}<0.001$ compared with internal group.

ening the potential of bufalin to be exploited as a therapeutic agent in the therapy of liver cancer (Fig. 2).

In the cell cycle analysis, Huh7, Hep3B and HA22T hepatoma cells were treated with bufalin and the alterations of cell cycle distributions by flow cytometry aided with propidium iodide staining were investigated. Bufalin did not induce the typical sub-G1, a close indicator for programmed cell death. Instead, bufalin increased the $\mathrm{G} 2 / \mathrm{M}$ phase accumulation time-dependently in all three cell types, suggesting G2/M phase arrest and a non-apoptotic mechanism (Fig. 3). The alterations of $\mathrm{G} 2 / \mathrm{M}$ phase markers, such as cdc25C, cyclin B and $\operatorname{cdc} 2 / \mathrm{cdk} 1$, also demonstrated the similar results at their 
Table IV. PCR array of gene expression pattern modulated by bufalin in Huh7 cells.

\begin{tabular}{|c|c|c|}
\hline Gene name & Description & Fold Alternatio \\
\hline CXCR4 & Chemokine (C-X-C motif) receptor 4 & 11.63 \\
\hline TNF & Tumor necrosis factor & 7.67 \\
\hline IFNG & Interferon $\gamma$ & 4.72 \\
\hline IFNA2 & Interferon $\alpha 2$ & 3.51 \\
\hline GABARAPL1 & $\mathrm{GABA}(\mathrm{A})$ receptor-associated protein like 1 & 2.64 \\
\hline PIK3CG & Phosphoinositide-3-kinase, catalytic, $\gamma$ polypeptide & 2.23 \\
\hline RPS6KB1 & Ribosomal protein S6 kinase, 70-kDa, polypeptide 1 & 2.08 \\
\hline MAP1LC3A & Microtubule-associated protein 1 light chain $3 \alpha$ & 1.72 \\
\hline SNCA & Synuclein $\alpha$ (non-A4 component of amyloid precursor) & 1.72 \\
\hline NFKB1 & Nuclear factor of $\kappa$ light polypeptide gene enhancer in B-cells 1 & 1.69 \\
\hline MAP1LC3B & Microtubule-associated protein 1 light chain $3 \beta$ & 1.68 \\
\hline UVRAG & UV radiation resistance associated gene & 1.66 \\
\hline BCL2L1 & BCL2-like 1 & 1.56 \\
\hline PRKAA1 & Protein kinase, AMP-activated, $\alpha 1$ catalytic subunit & 1.56 \\
\hline DRAM1 & DNA-damage regulated autophagy modulator 1 & 1.51 \\
\hline EIF2AK3 & Eukaryotic translation initiation factor $2-\alpha$ kinase 3 & 1.48 \\
\hline CTSS & Cathepsin S & 1.48 \\
\hline APP & Amyloid $\beta$ (A4) precursor protein & 1.47 \\
\hline INS & Insulin & 1.46 \\
\hline TGFB1 & Transforming growth factor $\beta 1$ & 1.36 \\
\hline ATG12 & ATG12 autophagy related 12 homolog (S. cerevisiae) & 1.36 \\
\hline PRKAA2 & Protein kinase, AMP-activated, $\alpha 2$ catalytic subunit & 1.33 \\
\hline ATG4A & ATG4 autophagy related 4 homolog A (S. cerevisiae) & 1.32 \\
\hline ATG9A & ATG9 autophagy related 9 homolog A (S. cerevisiae) & 1.26 \\
\hline GABARAPL2 & $\mathrm{GABA}(\mathrm{A})$ receptor-associated protein-like 2 & 1.24 \\
\hline ATG4D & ATG4 autophagy related 4 homolog D (S. cerevisiae) & 1.23 \\
\hline BECN1 & Beclin-1, autophagy related & 1.16 \\
\hline ATG16L1 & ATG16 autophagy related 16-like 1 (S. cerevisiae) & 1.13 \\
\hline MAPK8 & Mitogen-activated protein kinase 8 & 1.13 \\
\hline TGM2 & Transglutaminase 2 (C polypeptide, protein-glutamine- $\gamma$-glutamyltransferase) & 1.10 \\
\hline CTSB & Cathepsin B & 1.04 \\
\hline BAK1 & BCL2-antagonist/killer 1 & 1.04 \\
\hline AMBRA1 & Autophagy/beclin-1 regulator 1 & 1.01 \\
\hline GABARAP & $\mathrm{GABA}(\mathrm{A})$ receptor-associated protein & -1.03 \\
\hline TMEM74 & Transmembrane protein 74 & -1.04 \\
\hline CDKN1B & Cyclin-dependent kinase inhibitor 1B (p27, Kip1) & -1.06 \\
\hline RAB24 & RAB24, member RAS oncogene family & -1.07 \\
\hline ATG16L2 & ATG16 autophagy related 16-like 2 (S. cerevisiae) & -1.08 \\
\hline MAPK14 & Mitogen-activated protein kinase 14 & -1.09 \\
\hline ATG5 & ATG5 autophagy related 5 homolog (S. cerevisiae) & -1.09 \\
\hline CASP8 & Caspase 8, apoptosis-related cysteine peptidase & -1.09 \\
\hline TP73 & Tumor protein $\mathrm{p} 73$ & -1.13 \\
\hline ARSA & Arylsulfatase A & -1.14 \\
\hline HGS & Hepatocyte growth factor-regulated tyrosine kinase substrate & -1.19 \\
\hline ATG3 & ATG3 autophagy related 3 homolog (S. cerevisiae) & -1.19 \\
\hline CASP3 & Caspase 3, apoptosis-related cysteine peptidase & -1.21 \\
\hline CDKN2A & Cyclin-dependent kinase inhibitor 2A (melanoma, p16, inhibits CDK4) & -1.22 \\
\hline FAM176A & Family with sequence similarity 176, member A & -1.23 \\
\hline SQSTM1 & Sequestosome 1 & -1.25 \\
\hline
\end{tabular}


Table IV. Continued.

\begin{tabular}{|c|c|c|}
\hline Gene name & Description & Fold alteration \\
\hline RB1 & Retinoblastoma 1 & -1.28 \\
\hline TP53 & Tumor protein p53 & -1.28 \\
\hline CLN3 & Ceroid-lipofuscinosis, neuronal 3 & -1.29 \\
\hline EIF4G1 & Eukaryotic translation initiation factor $4 \gamma 1$ & -1.32 \\
\hline HDAC1 & Histone deacetylase 1 & -1.33 \\
\hline ESR1 & Estrogen receptor 1 & -1.33 \\
\hline GAA & Glucosidase $\alpha$; acid & -1.34 \\
\hline IFNA4 & Interferon $\alpha 4$ & -1.34 \\
\hline FAS & Fas (TNF receptor superfamily, member 6) & -1.35 \\
\hline BCL2 & B-cell CLL/lymphoma 2 & -1.36 \\
\hline DRAM2 & DNA-damage regulated autophagy modulator 2 & -1.39 \\
\hline ATG4B & ATG4 autophagy related 4 homolog B (S. cerevisiae) & -1.39 \\
\hline PIK3C3 & Phosphoinositide-3-kinase, class 3 & -1.42 \\
\hline BAX & BCL2-associated X protein & -1.43 \\
\hline PIK3R4 & Phosphoinositide-3-kinase, regulatory subunit 4 & -1.44 \\
\hline IRGM & Immunity-related GTPase family, $\mathrm{M}$ & -1.44 \\
\hline ULK1 & Unc-51-like kinase 1 (C.elegans) & -1.46 \\
\hline AKT1 & V-akt murine thymoma viral oncogene homolog 1 & -1.58 \\
\hline DAPK1 & Death-associated protein kinase 1 & -1.55 \\
\hline ATG9B & ATG9 autophagy related 9 homolog B (S. cerevisiae) & -1.64 \\
\hline HTT & Huntingtin & -1.66 \\
\hline ATG7 & ATG7 autophagy related 7 homolog (S. cerevisiae) & -1.69 \\
\hline PTEN & Phosphatase and tensin homolog & -1.71 \\
\hline ATG10 & ATG10 autophagy related 10 homolog (S. cerevisiae) & -1.74 \\
\hline BAD & BCL2-associated agonist of cell death & -1.75 \\
\hline BNIP3 & BCL2/adenovirus E1B 19-kDa interacting protein 3 & -1.78 \\
\hline ATG4C & ATG4 autophagy related 4 homolog C (S. cerevisiae) & -1.82 \\
\hline FADD & Fas (TNFRSF6)-associated via death domain & -1.89 \\
\hline IGF1 & Insulin-like growth factor 1 (somatomedin C) & -2.00 \\
\hline BID & BH3 interacting domain death agonist & -2.03 \\
\hline RGS19 & Regulator of G-protein signaling 19 & -2.07 \\
\hline ULK2 & Unc-51-like kinase 2 (C. elegans) & -2.50 \\
\hline HSP90AA1 & Heat shock protein $90-\mathrm{kDa} \alpha$ (cytosolic), class A member 1 & -2.75 \\
\hline TNFSF10 & Tumor necrosis factor (ligand) superfamily, member 10 & -4.69 \\
\hline HSPA8 & Heat shock $70-\mathrm{kDa}$ protein 8 & -10.70 \\
\hline
\end{tabular}

Genes (84) regulated with bufalin treatment by PCR array. GAPDH was used as an internal comparing standard. In the first column, the abbreviated gene names are listed; second column briefly describes the function, family or known domains of the genes listed in the first column; third column displays the fold-change after 12-h bufalin treatment compared with sham-treated control group.

protein level (Fig. 4). Consistent to our findings in hepatoma cells, mounting reports have shown that bufalin can arrest the cell cycle of gastric cancer cells, leukemia cells, bladder carcinoma cells at $\mathrm{G} 2 / \mathrm{M}$ phase (22-25).

Although the cell cycle arrest at G2/M phase and the mechanisms of bufalin-induced apoptosis (type I cell death) were investigated by several previous studies (21-26), but the autophagy program (type II cell death) induced by bufalin in human cancer cells has very few reports $(12,27)$. We were interested in revealing whether bufalin induced cell death via apoptosis or autophagy after cell cycle arrest. In literature, bufalin induced apoptosis via Fas through caspase- 3 and -8 and increased the level of cleaved-PARP in hepatoma cancer cells and prostate cancer cells, upregulating the expression of downstream Bax in vitro and in vivo $(10,28,29)$. In our study, however, we did not find increase in cytochrome $\mathrm{C}$ and PARP during bufalin treatment in Huh7 cells (Fig. 4). Instead, the mRNA levels of Fas, caspase- 3 and -8 and Bax was decreased (Table IV). These results may indicate that bufalin-induced cell death is not by the typical apoptosis pattern in Huh7 


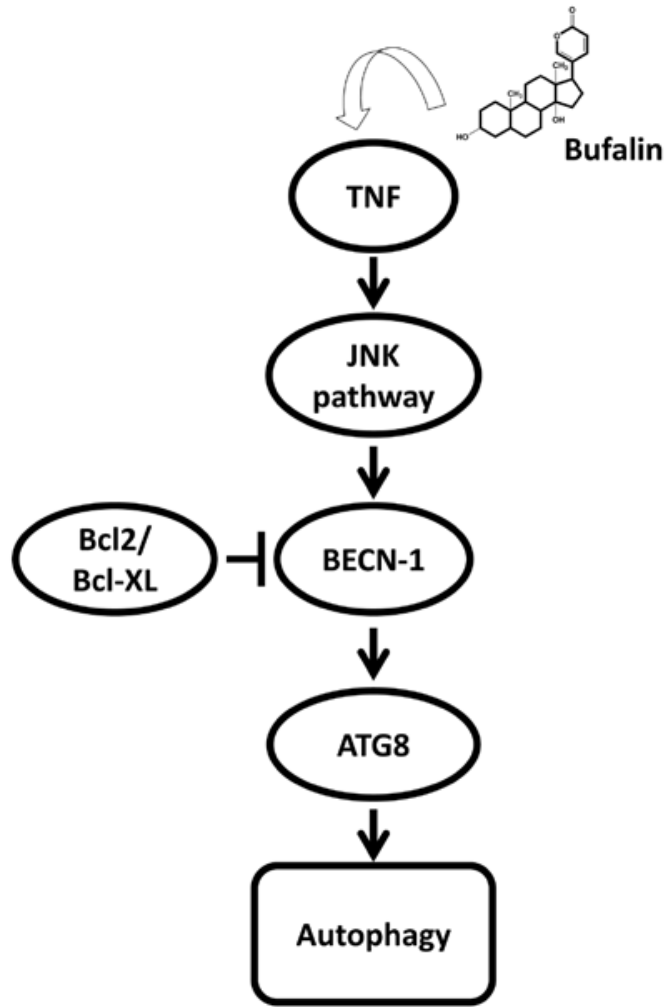

Figure 7. A proposed model of bufalin induced autophagy in hepatoma cells. This model summarizes the findings in this study, showing that bufalin induced autophagy was mainly via the regulation of TNF, JNK pathway, BECN-1 and the ATG8 novel pathway.

cells. Many anticancer agents, including fangchinoline and berberine, have been reported to induce autophagy without activation of caspase-dependent apoptosis $(30,31)$. Bid, Bcl-2 and Beclin-1 (Vps30/Atg6), were reported to be specific regulators for apoptosis and autophagy, which could determine the cell fate of apoptosis or autophagy (32-35). In our results, decreased Bcl-2 and increased Beclin-1 was found in the PCR array (Table IV). Bid was found to decrease at the mRNA level time-dependently in bufalin-treated Huh7, Hep3B and HA22T cells (Table I and Fig. 5). The protein level of Bid was also found to decrease time-dependently in Huh7 cells (Fig. 6). The above evidence collectively suggested that Bid, Bcl-2 and Beclin-1 may play the role in shifting the bufalin-treated hepatoma cells to undergo autophagy instead of apoptosis. Specific cytokines such as IFNA2/IFNA and IFNG/interferon $\gamma$, were reported to be important modulating factors to induce autophagy of the cells in interferon resistant bladder cancer and osteoposis (36,37). In our data collected from PCR array and real-time PCR-time course (Table I, Table II and Fig. 5), the increased mRNA levels of IFNA2/IFNA and IFNG/interferon $\gamma$ in Huh7 cells supported that autophagy was indeed induced by bufalin treatment in hepatoma cells.

After knowing that bufalin induced cell death of hepatoma cells was mainly by autophagy but not apoptosis, we are interested to reveal the mechanisms of bufalin-induced autophagy in hepatoma cells. Autophagic cell death has been reported to associate with the alterations in class III PI3K, ROS and the activation of JNK signaling pathway (38-40). However, our results (Table IV) showed that PIK3C3 (Vps34), a kinase in charge of triggering the class III PIK3 pathway, was decreased in its mRNA level in bufalin-treated Huh7 cells. The data suggested that the class III PIK3 pathway seems not to involve in bufalin-induced autophagy, even in the case that the mRNA level of Beclin-1, which was essential for Vps34 activation was indeed increased. Our results showed that TNF $\alpha / T N F$, JNK/ MAPK8, beclin-1 (BECN-1) and ATG8 family (GABARAP/ ATG8A, GABARAPL1/ATG8B, GABARAPL2/ATG8C, MAP1LC3A/ATG8E, MAP1LC3B/ATG8F) were activated in PCR array (TNF, MAPK8, BECN-1 and ATG8 in Table IV), in real-time PCR (TNF and GABARAPL1 in Table I, Table II and Fig. 5) and in western blotting (GABARAP and GABARAPL1 in Fig. 6). GABARAP and GABARAPL1 (ATG8A and ATG8B) interacted with the GABA receptor and high homology sequences with gabarap gene (41). Both of the proteins participate in the autophagy together with other members (LC3, GATE-16 and ATG8). In our data, both GABARAP and GABARAPL1 were highly expressed during bufalin treatment at transcriptional and translational levels. These findings supported that the JNK pathway was involved in bufalin-induced autophagy. Kawazoe et al found that the JNK pathway is one of the signaling pathways involved in bufalin-induced apoptosis in leukemia U937 cells (42). In this study, we have proven that the JNK pathway was also associated with bufalin-induced autophagy in human hepatoma cells. Consistent with our findings, JNK-mediated upregulation of Beclin-1 and ATG8 was found to play a causal role in autophagy-mediated cell death (Fig. 7) $(43,44)$.

The search for useful chemotherapy agents is valuable for treatment of hepatocellular carcinoma. To our knowledge, this is the first study to report on hepatoma cells that could be effectively killed by bufalin associated with cell cycle arrest at G2/M phase and through autophagy, not apoptosis. The JNK pathway was demonstrated to be mainly involved in the bufalin-induced autophagy in the hepatoma cells we investigated. Our study provides a platform for potential antihepatoma drug screening and mechanism determination. Bufalin was found to induce autophagy in hepatoma cells and to show potential for further pre-clinical investigations.

\section{Acknowledgements}

This study was supported by grants from China Medical University Hospital, Taichung, Taiwan (DMR-96-085).

\section{References}

1. Jemal A, Bray F, Center MM, Ferlay J, Ward E and Forman D: Global cancer statistics. CA Cancer J Clin 61: 69-90, 2011.

2. Forner A, Llovet JM and Bruix J: Hepatocellular carcinoma. Lancet 379: 1245-1255, 2012.

3. Eheman C, Henley SJ, Ballard-Barbash R, et al: Annual Report to the Nation on the status of cancer, 1975-2008, featuring cancers associated with excess weight and lack of sufficient physical activity. Cancer 118: 2338-2366, 2012.

4. Saika K and Matsuda T: Time trends in liver cancer mortality (1980-2008) in Japan, the USA and Europe. Jpn J Clin Oncol 42: 84, 2012.

5. Cao H, Phan H and Yang LX: Improved chemotherapy for hepatocellular carcinoma. Anticancer Res 32: 1379-1386, 2012.

6. Zhang S, Doudican NA, Quay E and Orlow SJ: Fluvastatin enhances sorafenib cytotoxicity in melanoma cells via modulation of AKT and JNK signaling pathways. Anticancer Res 31: 3259-3265, 2011 
7. Hagman M, Hayes RA, Capon RJ and Shine R: Alarm cues experienced by cane toad tadpoles affect post-metamorphic morphology and chemical defences. Funct Ecol 23: 126-132, 2009.

8. Gomes A, Bhattacharjee P, Mishra R, Biswas AK, Dasgupta SC and Giri B: Anticancer potential of animal venoms and toxins. Indian J Exp Biol 48: 93-103, 2010.

9. Gao H, Popescu R, Kopp B and Wang Z: Bufadienolides and their antitumor activity. Nat Prod Rep 28: 953-969, 2011.

10. Qi F, Inagaki Y, Gao B, et al: Bufalin and cinobufagin induce apoptosis of human hepatocellular carcinoma cells via Fas- and mitochondria-mediated pathways. Cancer Sci 102: 951-958, 2011.

11. Qi F, Li A, Inagaki Y, et al: Antitumor activity of extracts and compounds from the skin of the toad Bufo bufo gargarizans Cantor. Int Immunopharmacol 11: 342-349, 2011.

12. Xie CM, Chan WY, Yu S, Zhao J and Cheng CH: Bufalin induces autophagy-mediated cell death in human colon cancer cells through reactive oxygen species generation and JNK activation. Free Radic Biol Med 51: 1365-1375, 2011.

13. Gao Y, Li HX, Xu LT, et al: Bufalin enhances the anti-proliferative effect of sorafenib on human hepatocellular carcinoma cells through downregulation of ERK. Mol Biol Rep 39: 1683-1689, 2012.

14. Chen A, Yu J, Zhang L, et al: Microarray and biochemica analysis of bufalin-induced apoptosis of HL-60 cells. Biotechnol Lett 31: 487-494, 2009.

15. Takai N, Ueda T, Nishida M, Nasu K and Narahara H: Bufalin induces growth inhibition, cell cycle arrest and apoptosis in human endometrial and ovarian cancer cells. Int J Mol Med 21: 637-643, 2008

16. Berry DL and Baehrecke EH: Growth arrest and autophagy are required for salivary gland cell degradation in Drosophila. Cell 131: 1137-1148, 2007.

17. Cuervo AM and Macian F: Autophagy, nutrition and immunology. Mol Aspects Med 33: 2-13, 2012.

18. Janku F, McConkey DJ, Hong DS and Kurzrock R: Autophagy as a target for anticancer therapy. Nat Rev Clin Oncol 8: 528-539, 2011.

19. Liu YL, Yang PM, Shun CT, Wu MS, Weng JR and Chen CC: Autophagy potentiates the anti-cancer effects of the histone deacetylase inhibitors in hepatocellular carcinoma. Autophagy 6: 1057-1065, 2010.

20. Hsu CM, Hsu YA, Tsai Y, et al: Emodin inhibits the growth of hepatoma cells: finding the common anti-cancer pathway using Huh7, Hep3B and HepG2 cells. Biochem Biophys Res Commun 392: 473-478, 2010.

21. Takai N, Kira N, Ishii T, et al: Bufalin, a traditional oriental medicine, induces apoptosis in human cancer cells. Asian Pac J Cancer Prev 13: 399-402, 2012.

22. Li D, Qu X, Hou K, et al: PI3K/Akt is involved in bufalin-induced apoptosis in gastric cancer cells. Anticancer Drugs 20: 59-64, 2009

23. Numazawa $\mathrm{S}$, Shinoki MA, Ito $\mathrm{H}$, Yoshida $\mathrm{T}$ and Kuroiwa $\mathrm{Y}$ Involvement of $\mathrm{Na}+\mathrm{K}(+)$-ATPase inhibition in $\mathrm{K} 562$ cell differentiation induced by bufalin. J Cell Physiol 160: 113-120, 1994

24. Jing Y, Watabe M, Hashimoto S, Nakajo S and Nakaya K: Cell cycle arrest and protein kinase modulating effect of bufalin on human leukemia ML1 cells. Anticancer Res 14: 1193-1198, 1994

25. Hong SH and Choi YH: Bufalin induces apoptosis through activation of both the intrinsic and extrinsic pathways in human bladder cancer cells. Oncol Rep 27: 114-120, 2012

26. Zhu Z, Sun H, Ma G, Wang Z, Li E and Liu Y: Bufalin induces lung cancer cell apoptosis via the inhibition of PI3K/Akt pathway. Int J Mol Sci 13: 2025-2035, 2012.
27. Tsai SC, Yang JS, Peng SF, et al: Bufalin increases sensitivity to AKT/mTOR-induced autophagic cell death in SK-HEP-1 human hepatocellular carcinoma cells. Int J Oncol 41: 14311442,2012

28. Han KQ, Huang G, Gu W, Su YH, Huang XQ and Ling CQ: Anti-tumor activities and apoptosis-regulated mechanisms of bufalin on the orthotopic transplantation tumor model of human hepatocellular carcinoma in nude mice. World J Gastroenterol 13: 3374-3379, 2007.

29. Yu CH, Kan SF, Pu HF, Jea Chien E and Wang PS: Apoptotic signaling in bufalin- and cinobufagin-treated androgen-dependent and -independent human prostate cancer cells. Cancer Sci 99: 2467-2476, 2008.

30. Wang N, Pan W, Zhu M, et al: Fangchinoline induces autophagic cell death via p53/sestrin2/AMPK signalling in human hepatocellular carcinoma cells. Br J Pharmacol 164: 731-742, 2011.

31. Wang N, Feng Y, Zhu M, et al: Berberine induces autophagic cell death and mitochondrial apoptosis in liver cancer cells: the cellular mechanism. J Cell Biochem 111: 1426-1436, 2010

32. Pattingre S, Tassa A, Qu X, et al: Bcl-2 antiapoptotic protein inhibit Beclin 1-dependent autophagy. Cell 122: 927-939, 2005.

33. Motyl T, Gajkowska B, Zarzynska J, Gajewska M and Lamparska-Przybysz M: Apoptosis and autophagy in mammary gland remodeling and breast cancer chemotherapy. J Physiol Pharmacol 57 (Suppl 7): 17-32, 2006.

34. Zhang DM, Liu JS, Tang MK, et al: Bufotalin from Venenum Bufonis inhibits growth of multidrug resistant HepG2 cells through G(2)/M cell cycle arrest and apoptosis. Eur J Pharmacol 692: 19-28, 2012.

35. Trejo-Solis C, Jimenez-Farfan D, Rodriguez-Enriquez $\mathrm{S}$, et al: Copper compound induces autophagy and apoptosis of glioma cells by reactive oxygen species and jnk activation. BMC Cancer 12: 156,2012

36. Zhang XQ, Dunner K Jr and Benedict WF: Autophagy is induced by adenoviral-mediated interferon alpha treatment in interferon resistant bladder cancer and normal urothelial cells as a cell death protective mechanism but not by the bystander factors produced. Cancer Gene Ther 17: 579-584, 2010.

37. Zhang L, Guo YF, Liu YZ, et al: Pathway-based genome-wide association analysis identified the importance of regulation-ofautophagy pathway for ultradistal radius BMD. J Bone Miner Res 25: 1572-1580, 2010.

38. Gao M, Yeh PY, Lu YS, et al: OSU-03012, a novel celecoxib derivative, induces reactive oxygen species-related autophagy in hepatocellular carcinoma. Cancer Res 68: 9348-9357, 2008.

39. Borsello T, Croquelois K, Hornung JP and Clarke PG: N-methyld-aspartate-triggered neuronal death in organotypic hippocampal cultures is endocytic, autophagic and mediated by the c-Jun N-terminal kinase pathway. Eur J Neurosci 18: 473-485, 2003.

40. Tanida I: Autophagosome formation and molecular mechanism of autophagy. Antioxid Redox Signal 14: 2201-2214, 2011.

41. Chen C, Wang Y, Huang P and Liu-Chen LY: Effects of C-terminal modifications of GEC1 protein and gamma-aminobutyric acid type A (GABA(A)) receptor-associated protein (GABARAP), two microtubule-associated proteins, on kappa opioid receptor expression. J Biol Chem 286: 15106-15115, 2011.

42. Kawazoe N, Watabe M, Masuda Y, Nakajo S and Nakaya K: Tiam1 is involved in the regulation of bufalin-induced apoptosis in human leukemia cells. Oncogene 18: 2413-2421, 1999.

43. Li DD, Wang LL, Deng R, et al: The pivotal role of c-Jun NH2-terminal kinase-mediated Beclin 1 expression during anticancer agents-induced autophagy in cancer cells. Oncogene 28: 886-898, 2009

44. Byun JY, Yoon CH, An S, et al: The Rac1/MKK7/JNK pathway signals upregulation of Atg5 and subsequent autophagic cell death in response to oncogenic Ras. Carcinogenesis 30: 1880-1888, 2009. 